\section{Sammeln Sie CME-Punkte!}

Auch 2016 haben wir wieder ausgewählte CME-Kurse für Ihre hausärztliche Fortbildung in der Sommerakademie von Springer Medizin zusammengestellt.

Nutzen Sie die Sommerzeit, um reichlich CME-Punkte zu sammeln und um sich schnell und effizient fortzubilden!

Sichern Sie sich Ihre Punkte online

in der Springer Medizin Sommerakademie: www.springermedizin.de/sommerakademie

Die Teilnahme ist mit e.Med oder dem Zeitschriftenabo möglich.

Mit e.Med haben Sie nicht nur Zugriff auf die aktuelle Sommerakademie, Sie können das komplette Angebot von Springer Medizin nutzen. Sie haben Zugriff auf alle Inhalte von springermedizin.de, können alle Fachzeitschriften inklusive Archive im Volltext online lesen - zusätzlich erhalten Sie eine gedruckte Fachzeitschrift Ihrer Wahl nach Hause.

Vor allem erhalten Sie Zugang zu allen CME-Fortbildungen:

Mit rund 600 CME-zertifizierten Fortbildungskursen ist das Lernportal von Springer Medizin Deutschlands erste Adresse für Online-CME-Fortbildung. Sie haben außerdem die Möglichkeit, uneingeschränkt in unseren vielen englischsprachigen Journals zu recherchieren.

e.Med 30 Tage kostenlos und unverbindlich testen: www.springermedizin.de/eMed

Unser Kundenservice steht Ihnen wochentags (9-17 Uhr) kostenlos zur Verfügung: Tel. 08007780 777/ kundenservice@springermedizin.de

Ihre CME-Redaktion

\section{Fortbilden mit der Sommerakademie}

Endlich Urlaub, endlich reisen - viele Sonnenhungrige nutzen die langen Ferien, um die ersehnte Auszeit in den Tropen oder Subtropen zu verbringen. Ärgerlich nur, wenn Montezumas Rache oder fiese Malaria-Mücken die Idylle stören. Wie Sie ungeliebten Mikroben schon im Vorfeld vorbeugen, lesen Sie ab S. 20. Außerdem können Sie natürlich auch dieses Jahr in der Sommerakademie kräftig CMEPunkte sammeln. Viel Spaß beim Lesen und Fortbilden!

Claudia Daniels Redakteurin

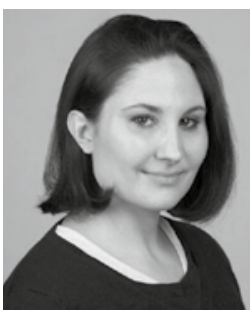

\title{
Pflicht zur Fortbildung vor Reha-Verordnung fällt weg
}

Die Vereinbarung von Qualitätssicherungsmaßnahmen zur Verordnung von Leistungen zur medizinischen Rehabilitation auf dem Muster 61 wird außer Kraft gesetzt. Hintergrund ist, dass zum 1. April 2016 die Rehabilitations-Richtlinie des Gemeinsamen Bundesausschusses (G-BA) geändert wurde und Vertragsärzte nun keine besonderen Qualifikationen mehr brauchen. Bisher mussten Hausärzte z. B. die fakultative Weiterbildung „Klinische Geriatrie“ absolvieren, ein Jahr in einer
Rehabilitationseinrichtung arbeiten oder eine Fortbildung von 16 Stunden über Grundlagen der International Classification of Functioning, Disability and Health (ICF) und die verordnungsfähigen RehaLeistungen absolvieren. Die Qualitätssicherungsvereinbarung war seinerzeit beschlossen worden, um die Qualifikationserfordernisse konkret auszugestalten. Sie definiert vor allem die Inhalte des Fortbildungskurses sowie die Anforderungen an den Kursleiter und die Referenten.

\section{Die Zukunft gehört dem E-Learning}

Dr. Max Kaplan, Vorsitzender des Deutschen Senats für ärztliche Fortbildung, sieht die Zukunft der strukturierten Fortbildung in E-Learning-Konzepten. Der Vorteil ist u.a. ein zeit- und ortsunabhängiger Zugang. „Wir werden die Präsenzveranstaltungen etwas zurückfahren zugunsten von $\mathrm{E}$ Learning, was in Flächenländern sehr hilfreich ist,“ sagte Kaplan im

Ge- spräch mit Springer Medizin. Dabei müsse man den Teilnehmern aber die Möglichkeit geben, die Inhalte mit einem Ansprechparntner $\mathrm{zu}$ reflektieren und $\mathrm{zu}$ evaluieren, betonte Kaplan.

Ein weiterer Trend ist laut Kaplan das Blended Learning. Hier absolvieren die Teilnehmer vorab eine E-Learning-Einheit, wodurch die Dauer der Präsenzveranstaltung deutlich verkürzt wird. Ein zusätzlicher Vorteil ist, dass die Teilnehmer die Veranstaltung mit einem gleichen Wissensstand besuchen und so effizienter und interaktiver arbeiten können.

\section{KV muss auf Kürzung hinweisen}

Vertragsärzten, die es nicht schaffen, innerhalb von fünf Jahren 250 Fortbildungspunkte nachzuweisen, drohen bekanntlich Honorarkürzungen. In den ersten vier Quartalen überweist die Kassenärztliche Vereinigung (KV) 10\% - danach sogar 25\% weniger Geld . Doch ganz automatisch geht das nicht, wie jetzt das Landessozialgericht Nordrhein-Westfalen klarstellte. Bevor sie das Geld einbehält, muss die KV eindeutig auf diese Gefahr hinweisen (Az.: L 11 KA 106/12).

Im konkreten Fall hatte ein Arzt im 2. Quartal 2011 rund 10.000 Euro weniger Honorar erhalten. Nach Darstellung der KV hatte der Sachbearbeiter den Arzt im Juni 2010 darauf hingewiesen, dass er nur noch bis April 2011 Zeit habe. Trotzdem seien die Fortbildungsnachweise nicht eingegangen.

Im Prozess argumentierte der Mediziner, dass er sich sehr wohl fortgebildet habe und man ihm nur vorwerfen könne, die Nachweise nicht rechtzeitig übersandt zu haben. Darauf aber hätte die KV ihn mindestens drei Monate vor Ablauf der Frist hinweisen müssen. Das Schreiben der KV von Juni 2010 habe er nicht erhalten. Dieser Einwand hatte vor Gericht Bestand, weil die KV die Zustellung des Schreibens nicht nachweisen konnte. 